\title{
EDITORIAL
}

\section{THANKS TO REVIEWERS OF PREVIOUS ISSUES:}

Umulker Ali Abdillahi
Anna Alvazzi del Frate
Szilvia Ádám
Piroska Balogh
Attila Bartha
Jack Birner
Julianna Bodó
Ágnes Czakó
Bruno Dallago
Zsuzsanna Elekes
Julianna Faludi
Menno Fenger
Anuska Ferligoj
Éva Fodor
István R. Gábor
Miklós Hadas
Max Haller
Balázs Hámori
József Hegedüs
Dezső Helmich
Mária Herczog
John Higley
Ildikó Hrubos
Ildikó Husz

Michal Illner

Gabriella Ilonszki

Csaba Jelinek

Pál Juhász

Gopalakrishnan

Karunanithi

Eszter Kelemen

Gábor Király

Márta Kiss

Bence Kollányi

Mariann Kopasz

András Körösényi

Zuza Kusa

Mihály Laki

David Lane

József Péter Martin

Attila Melegh

Richard Münch

Beáta Nagy

Ildikó Nagy

Attila Pilinszki

Anthony Oberscall

Emelka Obioha

Richard Ochanda
Antal Örkény

Árpád Rab

Andrew Ryder

Dumitru Sandu

Adriano Scribano

András Simonovits

Neil Smelser

Attila Károly Soós

Laura Szabó

Eleonóra Szanyi

Levente Székely

Judit Takács

Károly Takács

Róbert Tardos

Gábor Tóka

Kinga Tóth-Szabó

István György Tóth

Eric M. Uslaner

Adrienn Újhelyi

Szabolcs Varga

Balázs Vedres

Lilla Vicsek

Natalie Waechter 